\title{
Erratum to: Multi-view feature selection and classification for Alzheimer's Disease Diagnosis
}

\author{
Mingxing Zhang ${ }^{1} \cdot$ Yang Yang ${ }^{1} \cdot$ Fumin Shen ${ }^{1} \cdot$ \\ Hanwang Zhang ${ }^{2}$ - Yuan Wang ${ }^{2}$
}

Published online: 16 August 2016

(C) Springer Science+Business Media New York 2016

\section{Erratum to: Multimed Tools Appl DOI 10.1007/s11042-015-3173-5}

The original version of this article did not include detailed statements and acknowledgments for the data the authors used in the article.

1. In page 3, footnote 1 (i.e., "http://www.loni.ucla.edu/ADNI") should read: "Data used in preparation of this article were obtained from the Alzheimer's Disease Neuroimaging Initiative (ADNI) database (adni.loni.usc.edu). As such, the investigators within the ADNI contributed to the design and implementation of ADNI and/or provided data but did not participate in analysis or writing of this report. A complete listing of ADNI investigators can be found at: "http://adni.loni.usc.edu/wp-content/uploads/how_to_apply/ADNI_ Acknowledgement_List.pdf”.

The online version of the original article can be found at http://dx.doi.org/10.1007/s11042-015-3173-5

Yang Yang

dlyyang@gmail.com

Mingxing Zhang

superstar_zhang@hotmail.com

Fumin Shen

fumin.shen@gmail.com

Hanwang Zhang

hanwangzhang@gmail.com

Yuan Wang

iseway@nus.edu.sg

1 University of Electronic Science and Technology of China, Chengdu, China

2 National University of Singapore, Singapore, Singapore 
2. In page 4, section 3, second paragraph, the sentence should read "In this paper, we use the Alzheimer's Disease Neuroimaging Initiative (ADNI) database (adni.loni.usc.edu) as our original data. The ADNI was launched in 2003 as a public-private partnership, led by Principal Investigator Michael W. Weiner, MD. The primary goal of ADNI has been to test whether serial magnetic resonance imaging (MRI), positron emission tomography (PET), other biological markers, and clinical and neuropsychological assessment can be combined to measure the progression of mild cognitive impairment (MCI) and early Alzheimer's disease (AD). We consider two view sources, which are MRI and PET data".

3. The following should be added to the Acknowledgements section: "In addition, data collection and sharing for this paper was funded by the Alzheimer's Disease Neuroimaging Initiative (ADNI) (National Institutes of Health Grant U01 AG024904) and DOD ADNI (Department of Defense award number W81XWH-12-2-0012). ADNI is funded by the National Institute on Aging, the National Institute of Biomedical Imaging and Bioengineering, and through generous contributions from the following: AbbVie, Alzheimer's Association; Alzheimer's Drug Discovery Foundation; Araclon Biotech; BioClinica, Inc.; Biogen; Bristol-Myers Squibb Company; CereSpir, Inc.; Eisai Inc.; Elan Pharmaceuticals, Inc.; Eli Lilly and Company; EuroImmun; F. Hoffmann-La Roche Ltd. and its affiliated company Genentech, Inc.; Fujirebio; GE Healthcare; IXICO Ltd.; Janssen Alzheimer Immunotherapy Research \& Development, LLC.; Johnson \& Johnson Pharmaceutical Research \& Development LLC.; Lumosity; Lundbeck; Merck \& Co., Inc.; Meso Scale Diagnostics, LLC.; NeuroRx Research; Neurotrack Technologies; Novartis Pharmaceuticals Corporation; Pfizer Inc.; Piramal Imaging; Servier; Takeda Pharmaceutical Company; and Transition Therapeutics. The Canadian Institutes of Health Research is providing funds to support ADNI clinical sites in Canada. Private sector contributions are facilitated by the Foundation for the National Institutes of Health (www.fnih.org). The grantee organization is the Northern California Institute for Research and Education, and the study is coordinated by the Alzheimer's Disease Cooperative Study at the University of California, San Diego. ADNI data are disseminated by the Laboratory for Neuro Imaging at the University of Southern California". 